\title{
Characteristics, stability and outcomes of the 2011 GOLD COPD groups in the ECLIPSE cohort
}

\author{
Alvar Agusti ${ }^{1}$, Lisa D. Edwards², Bartolomé Celli³, William MacNee ${ }^{4}$, \\ Peter M. A. Calverley ${ }^{5}$, Hana Müllerova ${ }^{6}$, David A. Lomas ${ }^{7}$, Emiel Wouters ${ }^{8}$, \\ Per Bakke ${ }^{9,10}$, Steve Rennard ${ }^{11}$, Courtney Crim², Bruce E. Miller ${ }^{12}$, \\ Harvey O. Coxson ${ }^{13}$, Julie C. Yates ${ }^{2}$, Ruth Tal-Singer ${ }^{12}$ and Jørgen Vestbo ${ }^{14,15}$ \\ on behalf of the ECLIPSE investigators ${ }^{16}$
}

Affiliations: ${ }^{1}$ Thorax Institute, Hospital Clinic, IDIBAPS, Universitat de Barcelona and CIBER Enfermedades Respiratorias, FISIB, Mallorca, Spain. ${ }^{2}$ GlaxoSmithKline, Research Triangle Park, NC, ${ }^{3}$ Dept of Respiratory Medicine; Brigham and Women's Hospital, Boston, MA, ${ }^{11}$ University of Nebraska Medical Center, Omaha, NE, and ${ }^{12}$ GlaxoSmithKline, King of Prussia, PA, USA. ${ }^{4}$ University of Edinburgh \& Royal Infirmary, Edinburgh, ${ }^{5} \mathrm{Dept}$ of Respiratory Medicine, University Hospital Aintree, Liverpool, 'Epidemiology, GlaxoSmithKline R\&D, Middlesex, ${ }^{7} \mathrm{Dept}$ of Medicine, University of Cambridge, Cambridge Institute for Medical Research, Cambridge, and ${ }^{15}$ Manchester Academic Health Sciences Centre, University of Manchester, Manchester, UK. ${ }^{8}$ Dept of Respiratory Medicine, Maastricht University Medical Centre, Maastricht, The Netherlands. ${ }^{9}$ Institute of Medicine, University of Bergen, Bergen, and ${ }^{10}$ Dept of Thoracic Medicine, Haukeland University Hospital, Bergen, Norway. ${ }^{13}$ Dept of Radiology, University of British Columbia, Vancouver General Hospital, Vancouver, BC, Canada. ${ }^{14}$ Dept of Respiratory Medicine, Odense University Hospital/University of Southern Denmark, Denmark. ${ }^{16}$ For a complete list of principal investigators and centres participating in ECLIPSE see Acknowledgements.

Correspondence: A. Agusti: Institut del Tòrax. Hospital Clínic, Universitat de Barcelona. Villarroel 170, Escala 3, Planta 5. 08036 Barcelona, Spain: E-mail: alvar.agustidaclinic.ub.es

ABSTRACT The 2011 Global Initiative for Chronic Obstructive Lung Disease (GOLD) classifies patients with chronic obstructive pulmonary disease (COPD) into four groups (A to D).

We explored the characteristics, stability and relationship to outcomes of these groups within the ECLIPSE study (Evaluation of COPD Longitudinally to Identify Predictive Surrogate End-points) $(n=2101)$.

Main results showed that: 1) these groups differed in several clinical, functional, imaging and biological characteristics in addition to those used for their own definition; 2) A and D groups were relatively stable over time, whereas groups $\mathrm{B}$ and $\mathrm{C}$ showed more temporal variability; 3 ) the risk of exacerbation over 3 years increased progressively from A to D, whereas that of hospitalisation and mortality were lowest in A, highest in $\mathrm{D}$ and intermediate and similar in $\mathrm{B}$ and $\mathrm{C}$, despite the former having milder airflow limitation. The prevalence of comorbidities and persistent systemic inflammation were highest in group B.

The different longitudinal behaviour of group A versus B and C versus D (each pair with similar forced expiratory volume in $1 \mathrm{~s}$ (FEV1) values supports the 2011 GOLD proposal of assessing COPD patients by more than FEV1 only. However the assumption that symptoms do not equate to risk appears to be naïve, as groups B and C carry equally poor clinical outcomes, though for different reasons.

@ERSpublications

Exploring distribution, characteristics, temporal stability and relationship with 2011 GOLD group outcomes in ECLIPSE http://ow.ly/mjJ8p

This article has supplementary material available from www.erj.ersjournals.com

Received: Dec 022012 | Accepted after revision: Feb 232013 | First published online: June 132013

Copyright @ERS 2013 


\section{Introduction}

Chronic obstructive pulmonary disease (COPD) is a heterogeneous disease, and the forced expiratory volume in the $1 \mathrm{~s}$ (FEV1) does not describe this complexity fully [1]. The recently released 2011 Global Initiative for Chronic Obstructive Lung Disease (GOLD) recommendations recognise this limitation and propose a novel multidimensional approach to assess COPD patients, which includes the level of symptoms (as assessed by either the modified Medical Research Council (mMRC) score or the chronic obstructive pulmonary disease (COPD) Assessment Test (CAT), the FEV1 value (expressed as a percentage of predicted value) and the individual history of previous exacerbations [2]. As a result, COPD patients can now be categorised and treated according to four groups (A, B, C and D) [2].

Very recently, three studies have used existing cohorts of COPD patients to explore different aspects of this new GOLD assessment proposal. HAN et al. [3] used the COPDGene cohort [4] to explore the influence of the instrument used to assess the level of symptoms (mMRC versus the St. George's Respiratory Questionnaire (SGRQ), as a surrogate for CAT) on group assignment and risk of exacerbations during follow-up. LANGE et al. [5] investigated the ability of the 2011 GOLD proposal to predict the clinical course of the disease in two Danish general population cohorts combined [5]. Finally, SoRIANo et al. [6] determined the distribution and validity of the four GOLD 2011 groups as predictors of mortality in eleven small Spanish cohorts merged for the analysis.

The ECLIPSE study(Evaluation of COPD Longitudinally to Identify Predictive Surrogate End-points) is a large, observational, longitudinal, multicentre, international study that includes a very detailed, clinical, functional imaging, and biological characterisation of COPD patients with a 3-year follow-up [1, 7]. Therefore, it offers a unique opportunity to reproduce some of these previous analyses and to explore other novel aspects of potential relevance. Specifically, in this study we used the ECLIPSE cohort to: 1) compare the 2007 and 2011 GOLD recommendations, in terms of patient distribution and assessment of disease severity; 2) characterise in detail the four 2011 GOLD groups; 3) explore their stability after a 3-year followup; and 4) investigate their relationship with clinical relevant outcomes (exacerbations, hospitalisations and mortality), and identify significant risk factors.

\section{Methods \\ Study design}

The design of the ECLIPSE study has been previously published [7]. In brief the ECLIPSE study is an observational, longitudinal, controlled study that recruited patients who mostly attended the outpatient clinics of secondary or tertiary care hospitals and, occasionally, primary care. After a baseline visit, participants were evaluated at 3 months, 6 months and then every 6 months for 3 years. ECLIPSE complies with the Declaration of Helsinki and Good Clinical Practice Guidelines, and has been approved by the ethics committees of the participating centres. All participants provided written informed consent.

\section{Population}

The original ECLIPSE cohort included 2164 patients with COPD, as previously published [1]. Since the mMRC dyspnoea score was missing in 62 patients and one patient had an unknown GOLD stage, the present analysis includes 2101 GOLD stage I-IV patients (97\% of the original ECLIPSE cohort) with full 2011GOLD data. These 2101 patients were categorised into one of four groups (A, B, C or D) according to the 2011 GOLD recommendations [2] using the mMRC score (the CAT had not been published [8] when the ECLIPSE study was designed [7]) and the FEV1 value, both of which were measured at recruitment, as well as the number of exacerbations retrospectively recorded from the year before inclusion in the ECLIPSE study.

\section{Measurements}

The methodology used in ECLIPSE has also been reported in detail elsewhere $[1,7]$. We used standardised questionnaires to record dyspnoea (mMRC), comorbidities (American Thoracic Society and Division of

Clinical trial: This study is registered at clinicaltrials.gov with the identifier NCT00292552.

Support statement: The study was sponsored by GlaxoSmithKline (GSK study code SCO104960). A Steering Committee and a Scientific Committee, comprising in total of 11 academics and six representatives of the sponsor, developed the original study design and concept, the plan for the current analyses, approved the statistical plan, had full access to the data, and was responsible for decisions with regard to publication. The study sponsor did not place any restrictions with regard to statements made in the final paper. Two authors of the current paper (AA and JV) are members of the Scientific Committee of GOLD and, as such, co-authors of the new 2011 GOLD recommendations (www.goldcopd.org). The current analysis was conducted after these recommendations were made public in November 2011.

Conflict of interest: Disclosures can be found alongside the online version of this article at www.erj.ersjournals.com 
Lung Disease of the National Institute of Health questionnaire (ATS-DLD-78-adult)), health-status (St. George's Respiratory Questionnaire for COPD patients (SGRQ-C)) and depression (Center for Epidemiological Studies Depression Scale (CES-D)) [1,7]. Nutritional status was assessed by the body mass (BMI) and fat-free mass indexes (FFMI) by bioelectrical impedance [7]. COPD exacerbations requiring treatment with antibiotics, oral corticosteroids and/or hospitalisation in the year prior to the study (and during follow-up) were also recorded. Spirometry and the 6-min walking distance (6MWD) were performed according to international guidelines $[9,10]$. Spirometric reference values were those of the European Community for Coal and Steel (ECCS) [11]. The BMI, airflow obstruction, dyspnoea, exercise capacity (BODE) index was calculated according to CeLLI et al. [12]. All subjects underwent a low-dose computed tomography (CT) scan of the chest acquired using multi-detector-row CT scanners (GE Healthcare or Siemens Healthcare), as described elsewhere [1]. Hospitalisations and all-cause mortality were recorded during a 3-year follow-up [13, 14]. Finally, the annual rate of FEV1 decline in each patient [15] and the proportion of patients with persistent systemic inflammation at baseline [14] were calculated as reported previously and included in the current analysis.

\section{Statistical analysis}

Results are shown as mean \pm SD or proportion, as appropriate. Jonckheere-Terpstra tests were used to assess differences in subject characteristics among groups with ordering of $A \leqslant B \leqslant C \leqslant D$ [16]. Kaplan-Meier curves were constructed to describe the occurrence of exacerbations, hospitalisations and all-cause mortality over the study period for both the 2007 [17] and 2011 GOLD [2] classifications. Concordance probabilities and their associated $95 \%$ confidence intervals were calculated for Cox regression models for exacerbations, hospitalisations and all-cause mortality to evaluate the predictive accuracy of the 2007 and 2011 GOLD classifications [18]. A Cox proportional hazards regression model was used to identify factors associated with increased risk of mortality and hospitalisation, A p-value $<0.05$ (two sided) was considered statistically significant.

\section{Results \\ Patient distribution and assessment of disease severity by the 2007 and 2011 GOLD recommendations}

Figure 1 shows the distribution of the COPD patients included in the analysis according to the 2007 (fig 1a) and 2011 (fig 1b) GOLD recommendations. The latter resulted in a rightward shift of the distribution, indicating that more patients were being classified in more severe groups. The mean \pm SD 2007 GOLD stage was not different between groups A $(2.0 \pm 0.0)$ and B $(2.0 \pm 0.1)$, and only slightly worse (albeit statistically significantly $\mathrm{p}<0.001)$ between groups $\mathrm{C}(3.0 \pm 0.5)$ and $\mathrm{D}(3.2 \pm 0.6)$. Figure $1 \mathrm{c}$ also shows the cumulative distribution of the four 2011 GOLD groups within each 2007 GOLD stage. GOLD stage II includes a large proportion of group A (54\%) and B (32\%) patients but also some group C (7\%) and D patients $(8 \%)$. Since, by definition, GOLD stage II patients must have an FEV1 $\geqslant 50 \%$ pred [17], these latter two groups were classified as high risk groups ( $\mathrm{C}$ or $\mathrm{D}$ ) exclusively, because of their past exacerbation history ( $\geqslant 2$ per year.). Alternatively, also by definition, GOLD stages III and IV cannot include group A or B patients, where FEV1 must be $\geqslant 50 \%$ of reference [2]. There were more group D patients in GOLD stage IV than GOLD stage III (fig. 1c). The frequency distribution of these four groups was not different in males and females (online supplementary table $1 \mathrm{E}$ ).

\section{Characterisation of the 2011 GOLD groups}

The application of the 2011 GOLD assessment criteria resulted in the expected differences in the mMRC scores, FEV1 and exacerbation rates between the four groups (table 1). In addition, these four groups differed also in many of the other characteristics studied (tables 1 and online supplementary table 2E). It is of note that some variables were particularly abnormal in the two high symptoms groups (B and D; SGRQ$\mathrm{C}$ and CES-scores, many comorbid conditions, exercise capacity and most inflammatory biomarkers), whereas others were mostly abnormal in the two high risk groups ( $\mathrm{C}$ and $\mathrm{D}$; amount of emphysema present and arterial oxygenation saturation) (tables 1 and online supplementary table 2E). Finally, of note, age, gender, cumulative smoking exposure, FEV1 reversibility and the annual rate of FEV1 decline were either not different between groups or differences were of unlikely clinical relevance (tables 1 and online supplementary table 2E).

As in two previous studies $[4,5]$, we stratified the $\mathrm{C}$ and $\mathrm{D}$ groups further according to the specific criteria that determined the inclusion of patients in these high risk groups ( $\mathrm{FEV} 1<50 \%$ reference (C1 or D1), exacerbation history $\geqslant 2$ years. (C2 or D2) or both (C3 or D3)). An FEV1 $<50 \%$ of reference alone was the most frequent reason to classify a patient as high risk in either the $\mathrm{C}$ or $\mathrm{D}$ groups $(70 \%$ and $63 \%$ of cases, respectively), whereas the presence of frequent exacerbations alone was responsible for a much lower 

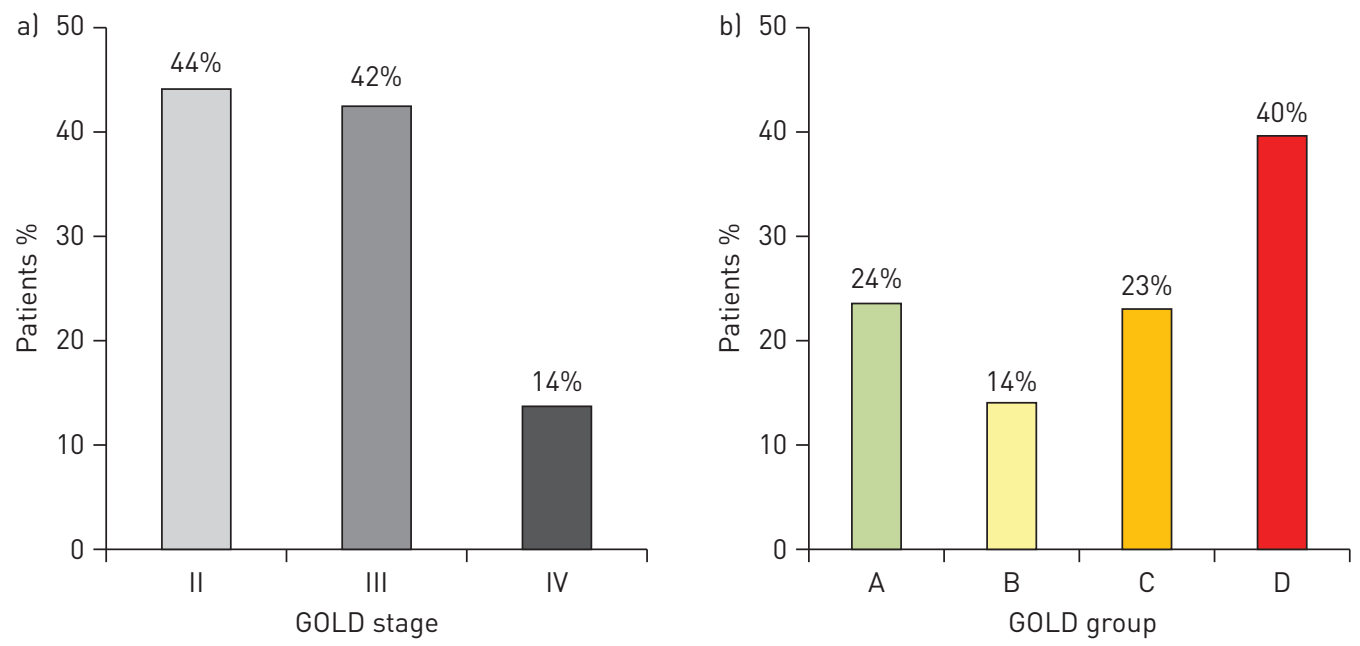

c)
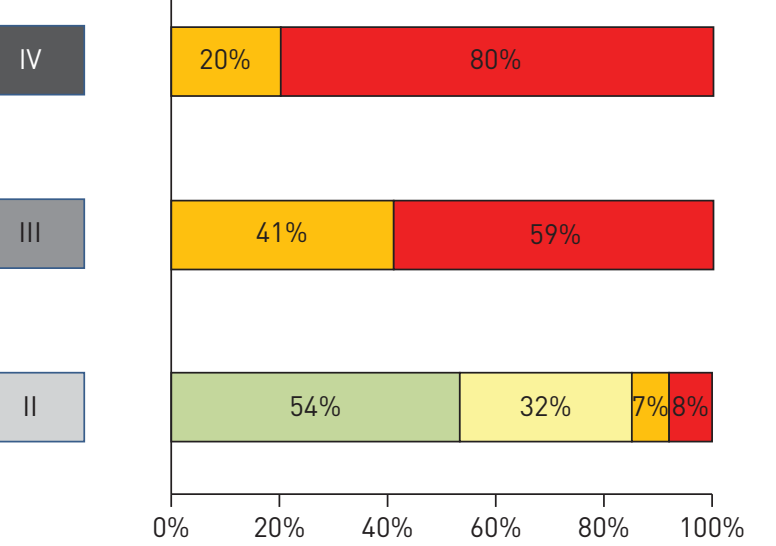

FIGURE 1 Frequency distribution of the chronic obstructive pulmonary patients studied here according to the 2007 (a) or 2011 (b) Global Initiative for Chronic Obstructive Lung Disease (GOLD) recommendations. Two subjects were classified as having GOLD stage I but were not included in a) or b). c) Shows the cumulative distribution of the four patient groups for the 2011 GOLD classification within each 2007 GOLD stage.

proportion of patients ( $13 \%$ and $9 \%$, respectively); the remaining patients ( $18 \%$ and $28 \%$, respectively) were classified as $\mathrm{C}$ or D patients because of the presence of both low FEV1 and frequent exacerbations. Online supplementary tables $3 \mathrm{E}$ and $4 \mathrm{E}$ present the main characteristics of these three $\mathrm{C}$ and $\mathrm{D}$ subgroups. By definition, exacerbation frequency was particularly high in C2/D2 and C3/D3.

\section{Temporal stability of the 2011 GOLD groups during the 3-year follow-up}

Figure 2 presents the distribution of the four 2011 GOLD groups both at recruitment and 3 years later. However, it is important to note that not all patients studied at recruitment contributed data during the 3year follow-up, due to a variety of reasons that include death, drop-out, lost for follow-up and/or missing data. This effect was less marked in group A, worse in group D and intermediate in groups B and C. With this caveat in mind, it is interesting to see that the relative majority of $\mathrm{A}$ and $\mathrm{D}$ patients do remain in their original classification, whereas B and C patients have greater temporal variability (fig. 2). B patients either remained in the same category (B), improved (A) or deteriorated (D) in roughly similar proportions. By contrast $\mathrm{C}$ patients, either remained stable (C) or deteriorated (D) and only a relatively small fraction improved ( $\mathrm{B}$ or A). Finally, it is of note that the proportion of patients that moved to group A from B, C or $\mathrm{D}$ was lowest in $\mathrm{D}$, intermediate in $\mathrm{C}$ and largest in $\mathrm{B}$ (fig. 2).

Relationship with long-term outcomes: comparison of the 2007 and 2011 GOLD recommendations Figure 3 presents Kaplan-Meier curves that describe the occurrence of exacerbations (a, d and g), hospitalisations (b, e and h) and all-cause mortality (c, f and i) over the study period for 2007 GOLD stages in all patients $(\mathrm{a}-\mathrm{c}), 2011$ GOLD groups in all patients $(\mathrm{d}-\mathrm{f})$ and 2011 GOLD groups for patients with a FEV $1<50 \%$ reference (GOLD II). Within 2007 GOLD stages, each of the pairwise comparisons are significantly different for exacerbations, hospitalisations $(\mathrm{p}<0.001)$ and all-cause mortality $(\mathrm{p} \leqslant 0.002)$. 
TABLE 1 Outcomes of the four groups of patients as per the 2011 Global Initiative for Chronic Obstructive Lung Disease (GOLD) recommendations [2].

\section{Distribution}

2011 GOLD groups

B C

C

D

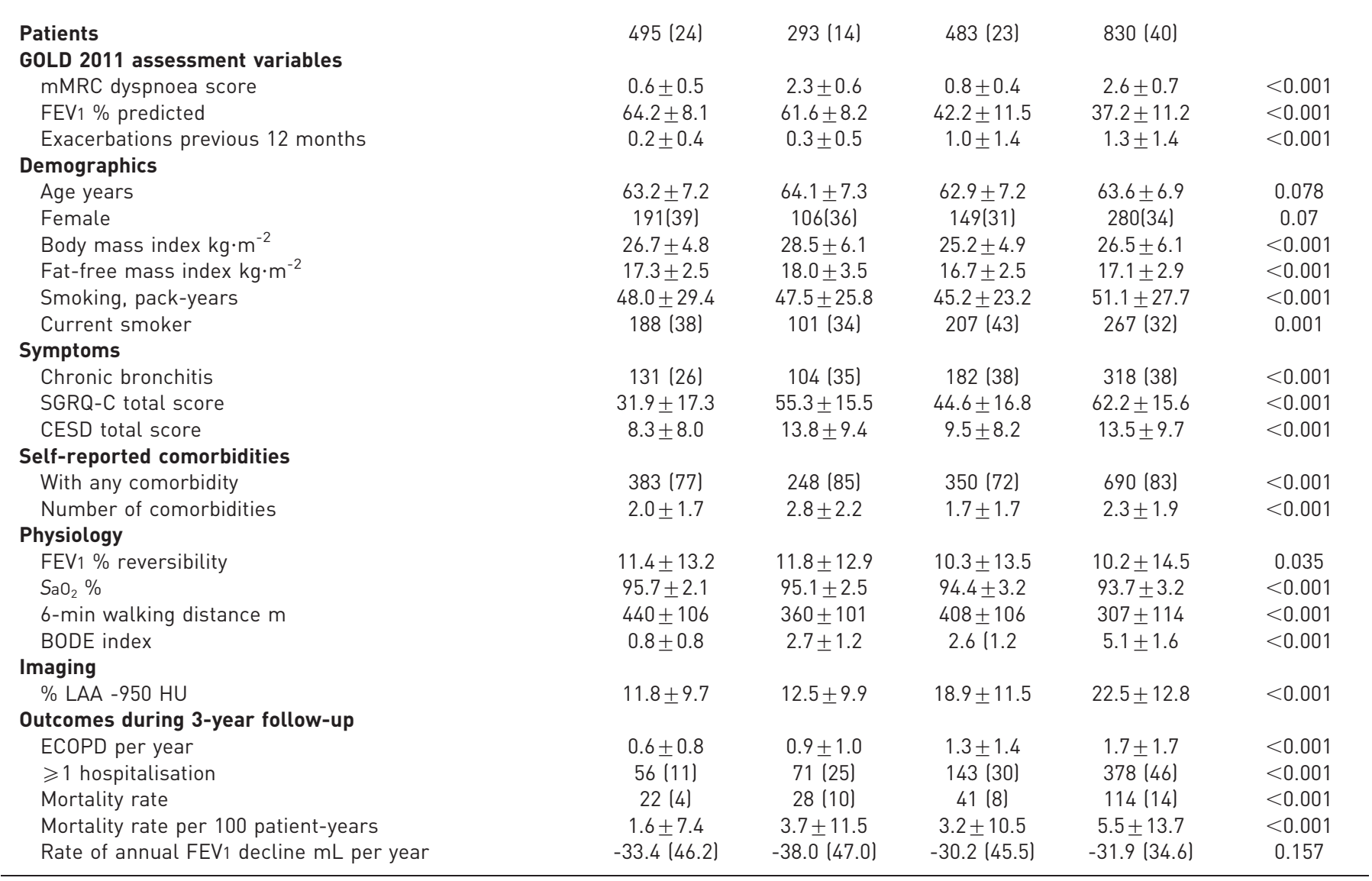

Data are presented as $\mathrm{n}(\%)$ or mean $\pm \mathrm{SD}$, unless otherwise stated. mMRC: dyspnoea score according to the modified Medical Research Council questionnaire; FEV1; forced expiratory volume in $1 \mathrm{~s}$; SGRQ-C: St George's Respiratory Questionnaire for chronic obstructive pulmonary disease (COPD) patients; CESD: Center for Epidemiological Studies Depression Scale; $\mathrm{SaO}_{2}$; arterial oxygen saturation; BODE: body mass index, airflow obstruction, dyspnoea, exercise capacity index; \% LAA: percentage of low attenuation areas (i.e. emphysema) in the computed tomography; ECOPD: exacerbation of COPD.

Similarly, within 2011 GOLD groups (all patients), each of the pairwise comparisons are also significantly different for exacerbations $(\mathrm{p}<0.001$ ), but not for hospitalisations or mortality, which were different in A and $\mathrm{D}(\mathrm{p} \leqslant 0.010)$, but similar in B and C patients, despite group B having better lung function and fewer exacerbations; yet, group B also had, at baseline, the highest prevalence of comorbidities (table 1), particularly cardiac comorbidities (online supplementary table $2 \mathrm{E}$ ), and of patients with persistent systemic inflammation (fig. 4), defined as we described previously [14].

Compared with the 2007 GOLD classification, the 2011 GOLD classification had a higher concordance probability (95\% CI) for predicting exacerbations (0.591 (0.577-0.605) versus 0.570 (0.558-0.583); $\mathrm{p}=0.003)$ and hospitalisations $(0.659(0.640-0.679)$ versus $0.628(0.610-0.646) ; \mathrm{p}=0.001)$; both classifications were similar for the prediction of all-cause mortality $(0.596(0.562-0.630)$ versus 0.614 $(0.576-0.653) ; \mathrm{p}=0.32)$.

Because there is limited information in the ECLIPSE database on specific causes of death [1,7], we used a Cox proportional hazards regression model to identify factors associated with increased risk of mortality. These included more severe airflow limitation, high age, low BMI, high FFMI, increased number of comorbidities, and lower exercise capacity (table 2). Interestingly, GOLD B patients had less airflow 


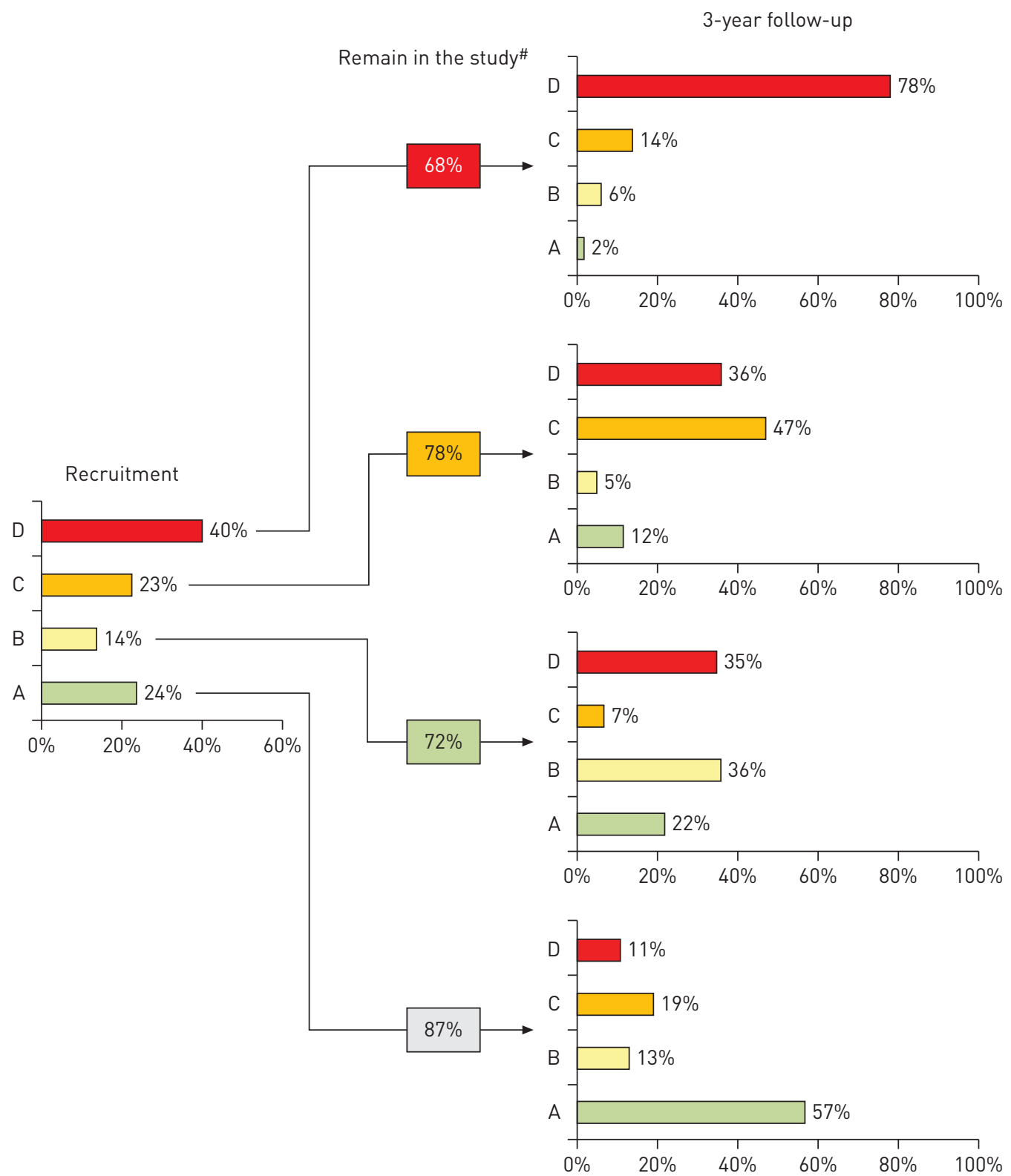

FIGURE 2 Temporal variation of the four 2011 Global Initiative for Chronic Obstructive Lung Disease groups at recruitment and during the 3 -year follow-up. Boxes with percentages indicate the proportion of baseline patients available for examination at the 3-year follow-up. ${ }^{*}$ : death/drop-out/lost for follow-up/missing data.

limitation and higher BMI than GOLD C patients, but were older, had a higher FFMI, more comorbidities, and poorer exercise capacity (table 1). These differences may contribute to their similar mortality during follow-up (fig. 3). A similar set of covariates (FEV1 \% pred, age, BMI, number of comorbidities, prior exacerbation history, arterial oxygen saturation and dyspnoea) were associated with increased risk of hospitalisation (table 2). For each of these covariates, differences were also noted between GOLD B and GOLD C patients (table 1).

Two final aspects of our analysis deserve comment. First, there were no significant differences between gender across the four groups for mortality or hospitalisation during follow up (online supplementary table $1 \mathrm{E})$ but females had a higher exacerbation rate than males $(\mathrm{p} \leqslant 0.017)$. Secondly, if only 2007 GOLD stage II patients (FEV1 $>50 \%$ of reference) are considered (fig. 1c), the 2011 GOLD proposal lacks discriminant power to predict exacerbations in C2 and D2 patients, the risk of hospitalisation is similar and intermediate in C2 and B patients and, interestingly, mortality during follow-up was zero in C2 patients (fig. 3g-i). 

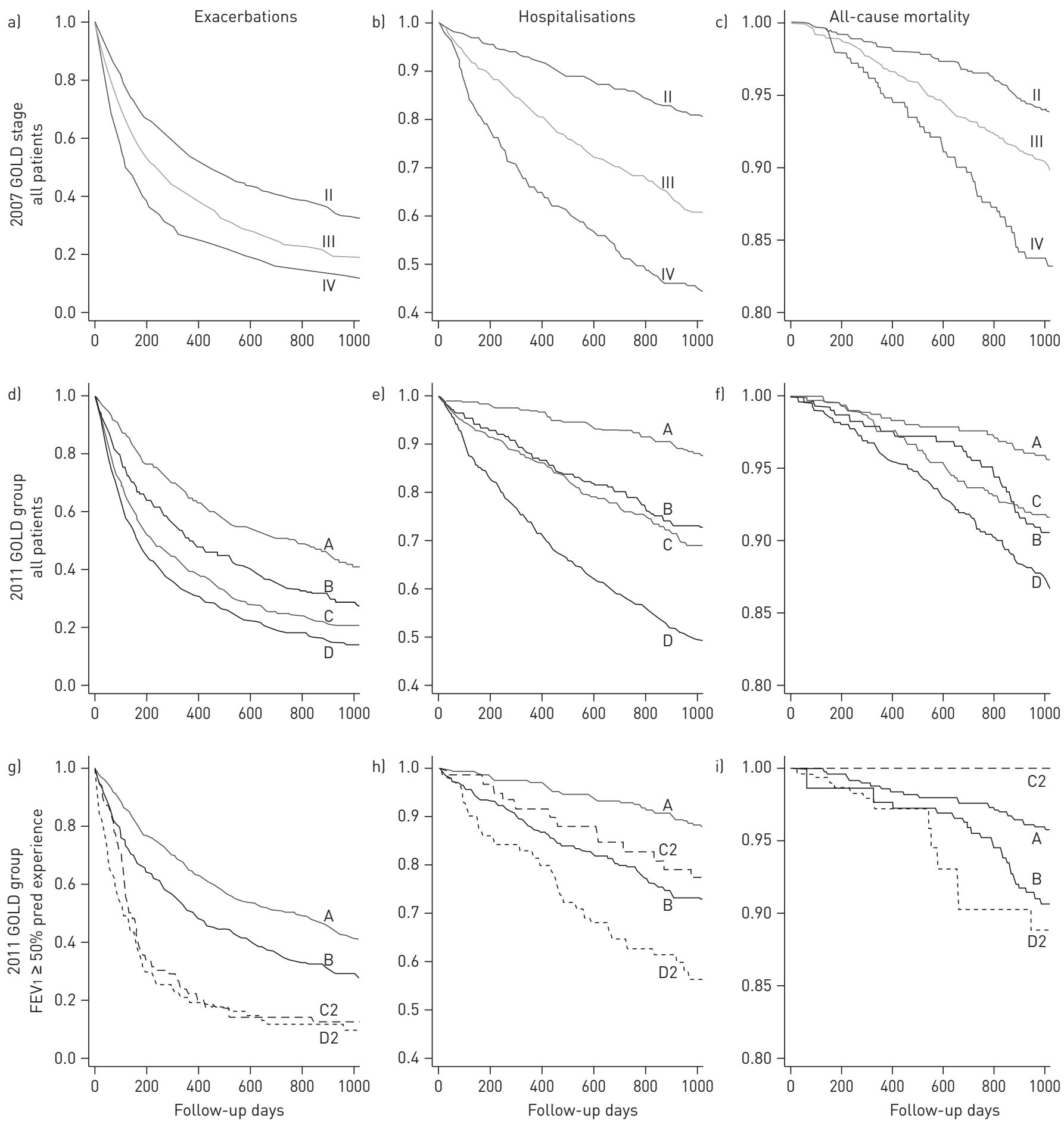

FIGURE 3 Kaplan-Meier curves for exacerbations (a, d and g), hospitalisations during follow-up (b, e and h) and all-cause mortality (c, f and i) for the 2007

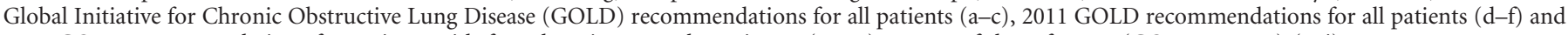
2011 GOLD recommendations for patients with forced expiratory volume in $1 \mathrm{~s}$ (FEV1) $\geqslant 50 \%$ of the reference (GOLD stage II) (g-i).

\section{Discussion}

The 2011 GOLD recommendations have proposed a novel, multidimensional strategy to assess disease severity and guide therapy in COPD [2]. Because this proposal was mostly based on expert opinion [2], several recent studies have used existing COPD cohorts to test it $[3,5,6]$. Our study confirms some of their results and extends them by presenting novel aspects not previously explored. 

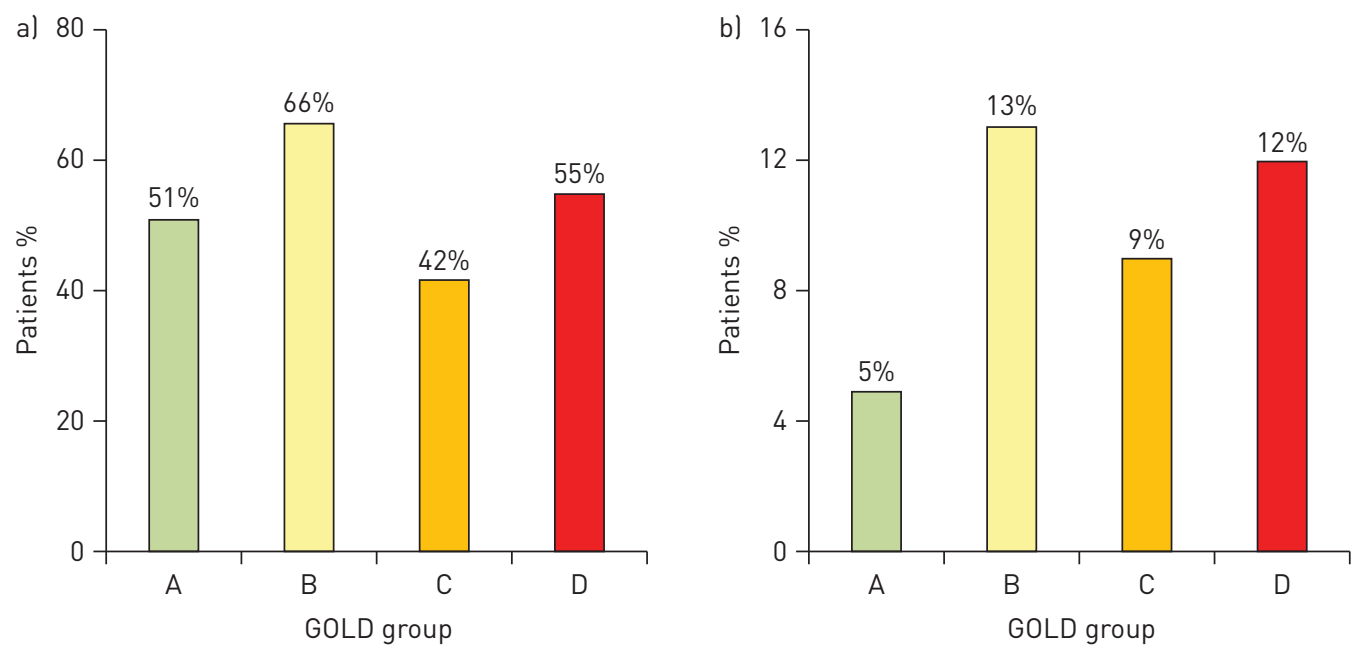

FIGURE 4 Percentage of patients with at least one self-reported comorbidity (a) or persistent, systemic inflammation (b), as described by AGUSTI et al. [14], in the four groups of the 2011 Global Initiative for Chronic Obstructive Lung Disease (GOLD) patient classification. a) $\mathrm{p}<0.05$ for all comparisons except for group A versus group B. b) $\mathrm{p}<0.05$ for all comparisons except for group B versus group D. It is of note that both were highest in B patients.

\section{Previous findings}

Our results reproduce and support five previous observations. 1) In keeping with LANGE et al. [5] we found that the new 2011 GOLD classification assesses disease severity differently from the previous 2007 proposal, since more individuals are categorised in the more severe group (fig. 1a and b). This is not surprising if the multidimensional nature of the 2011 GOLD proposal is considered [2]. 2) The prevalence of the four 2011 GOLD groups in our study (fig. 1b) was similar to that reported in previous COPD cohorts recruited from hospital clinics [3, 6], but clearly different from that described in COPD patients identified in the general population, where group A clearly predominates [5].3) In agreement with previous reports [3,5] we found that a low FEV1 ( $<50 \%$ reference) was the main reason for classifying patients as high risk (C and D groups). 4) We reproduced previous observations (fig 3d-f) indicating that the new 2011 GOLD assessment proposal predicts future exacerbations appropriately $[3,5]$. Likewise, in keeping with LANGE et al. [5], we

\section{TABLE 2 Cox proportional hazards regression analyses for all-cause mortality and hospitalisations during follow-up}

Independent variable

Mortality

Forced expiratory volume in $1 \mathrm{~s} \%$ predicted

Age years

Body mass index $\mathrm{kg} \cdot \mathrm{m}^{-2}$

Fat-free mass index $\mathrm{kg} \cdot \mathrm{m}^{-2}$

Number of comorbidities

6-min walking distance $\mathrm{m}$

Hospitalisations

Modified Medical Research Council dyspnoea score

Forced expiratory volume in $1 \mathrm{~s} \%$ predicted

Exacerbations in previous 12 months

Age years

Body mass index $\mathrm{kg} \cdot \mathrm{m}^{-2}$

Hypertension

Angina

Osteoarthritis

Diabetes

Depression requiring treatment

Number of comorbidities

Arterial oxygen saturation \%
Estimate

p-value

$\begin{array}{lc}0.982(0.969-0.996) & 0.011 \\ 1.052(1.021-1.085) & <0.001 \\ 0.931(0.882-0.983) & 0.01 \\ 1.146(1.036-1.267) & 0.008 \\ 1.100(1.001-1.210) & 0.048 \\ 0.997(0.996-0.999) & 0.002 \\ & \\ 1.246(1.119-1.387) & <0.001 \\ 0.974(0.966-0.982) & <0.001 \\ 1.195(1.123-1.270) & <0.001 \\ 1.021(1.005-1.038) & 0.01 \\ 0.979(0.960-0.999) & 0.04 \\ 0.669(0.526-0.851) & 0.001 \\ 0.565(0.371-0.858) & 0.007 \\ 0.695(0.489-0.987) & 0.042 \\ 0.604(0.392-0.932) & 0.023 \\ 0.657(0.473-0.912) & 0.012 \\ 1.209(1.113-1.314) & <0.001 \\ 0.929(0.902-0.956) & <0.001\end{array}$

Data are presented as hazard ratio (95\% confidence interval), unless otherwise stated. 
observed that mortality was similar in groups B and C (fig. 4), despite the former having, by definition, better lung function and fewer exacerbations [2]. We think that this is a clear example of how the new 2011 GOLD assessment proposal goes beyond FEV1 and identifies a group of patients who, despite the presence of moderate airflow limitation, are at high risk of mortality. SORIANO et al. [6] reported a similar observation over 3- but not at 10-year follow-up, raising the possibility of different causes of death at different timepoints during follow-up. The specific causes of death were not investigated by SoRIANO et al. [6], but LANGE et al. [5] reported that B patients often died of cardiovascular disease and cancer. In keeping with this observation, we also found that B patients showed the highest prevalence of comorbidities and included more patients with persistent systemic inflammation (fig. 4, table 1). The former is known to have a direct and significant impact on survival in COPD [19] and the latter has been recently shown to increase mortality six-fold, irrespective of the severity of the pulmonary abnormalities present [14]. These observations may have clinical implications for the practitioner, since B patients appear to be a unique group that deserve special attention in order to understand the origin of their greater symptoms, which are likely to relate to the presence of comorbidities, which may occur in patients with moderate airflow limitation [1], and need to be actively looked for and treated adequately if present [2]. 5) The observation that despite having frequent exacerbations and requiring frequent hospitalisations, $\mathrm{C} 2$ patients have a mortality of zero during follow-up (fig. 3) is also of clinical interest and in keeping with the observations by LANGE et al. [5].

\section{Novel observations}

Our study extends previous reports $[3,5,6]$ by providing potentially relevant information on six aspects not investigated before.1) We found that the four 2011 GOLD groups differed in many clinical, functional, imaging and biological characteristics in addition to those used for their categorisation (tables 1 and online supplementary table 2E). Interestingly, some of them (comorbidities and systemic inflammation) are more prevalent in the two high symptom groups (B and D), whereas others (emphysema and low arterial oxygen saturation) are more prevalent in the two high risk groups (C and D) (tables 1 and online supplementary table 2E). Although both HAN et al. [3] and LANGE et al. [5] identified some of these differences, the level of characterisation of the participants in ECLIPSE is much more detailed so, this information constitutes an important repository of data (tables 1 and online supplementary tables $2 \mathrm{E}, 3 \mathrm{E}$ and $4 \mathrm{E}$ ) of potential use for a better understanding of the disease and for the design of future studies. 2) Our analysis is the first to investigate the temporal stability of the four 2011 GOLD groups. We observed that a relative majority of A and $\mathrm{D}$ patients do actually remain in their original classification, whereas $\mathrm{B}$ and $\mathrm{C}$ patients present higher temporal variability (fig. 2). These changes can be due to either disease progression, or the beneficial effect of therapy but, in any case, this observation provides evidence of the degree of heterogeneity of the natural history of the disease and may highlight a potential window of opportunity for therapeutic intervention. 3 ) Also not explored previously $[3,5,6]$, we found that the 2011 GOLD assessment proposal does not separate the COPD cohort into groups that differ in their rate of FEV1 decline (table 1). This suggests that further segmentation of the COPD population beyond that proposed by 2011 GOLD assessment may be required to fully describe COPD heterogeneity, since several recent studies $[20,21]$, including an analysis of the ECLIPSE cohort [15], have clearly shown that the rate of FEV1 decline varies among COPD patients. 4) No previous study has investigated the relationship of the four 2011 GOLD groups with hospitalisations during follow-up. We found that, similarly to mortality, hospitalisations were less prevalent in A, most in D and intermediate and similar in the B and C groups (fig. 3). As in the case of mortality discussed above, this observation emphasises a, sometimes overlooked, fact; i.e. that patients with COPD may require hospitalisation (and/or die) for reasons not directly related to COPD (comorbidities). Because they are amenable to therapy [22], this observation supports the emphasis made by the 2011 GOLD document on the importance of actively looking for, and adequately treating if present, the comorbidities known to frequently occur in COPD patients [2]. 5) Since ECLIPSE does not have information on specific causes of death, we explored factors associated with increased risk of mortality (and hospitalisations) using a Cox proportional hazards regression model. Interestingly the identified risk factors were similar for mortality and hospitalisations (table 2). The fact that they were distributed relatively evenly between $\mathrm{B}$ and $\mathrm{C}$ patients (tables 1 and online supplementary table $2 \mathrm{E}$ ) may in part explain the relatively similar outcomes exhibited by these two groups despite their different severities of airflow limitation and/or past history of exacerbations, and may help clinicians to assess the risk of individual patients. 6) We explored, for the first time, the potential gender differences among 2011 GOLD groups. We found that their frequency distribution was not different in males or females (online supplementary table 1E). Furthermore, we observed that there were no significant gender differences for mortality or hospitalisations although, interestingly, females had a higher $(\mathrm{p} \leqslant 0.017)$ exacerbation rate during follow-up than males (online supplementary table $1 \mathrm{E}$ ). 


\section{Potential limitations}

The following potential limitations of our study deserve comment. 1) Like all previous studies [3, 5, 6], our analysis took advantage of an existing cohort assembled for different purposes, so it might not mirror the population of COPD patients at large. 2) Because patients enrolled in the ECLIPSE study were mostly recruited from hospital clinics and were treated according to the attending physician, our results may not be directly generalised to untreated patients or patients with milder disease (i.e. GOLD stage I). However, it is of interest that LANGE et al. [5] reported similar observations in patients recruited from the general population with a skewed distribution towards milder disease (77\% classified in the A group). 3) Because of the inclusion criteria used in the ECLIPSE study (age 40-75 years, baseline post-bronchodilator FEV1 $<80 \%$ of the predicted value, baseline post-bronchodilator FEV1/FVC $\leqslant 0.7$ and a smoking history of $\geqslant 10$ pack-years [7]), it is possible that a small fraction of A and B patients analysed here may be above the lower limit of the normal. Yet, all of them were diagnosed of COPD by their attending physicians considering their exposure to risk factors and current clinical symptomatology. 4) Comorbidities were self-reported and we lack information on specific causes of death, so some of our observations and interpretations require prospective validation in future studies.

\section{Conclusions}

The different longitudinal behaviour of group $\mathrm{A}$ versus $\mathrm{B}$, and $\mathrm{C}$ versus $\mathrm{D}$ (both pairs with similar FEV1 values) supports the 2011 GOLD proposal of assessing COPD patients by more than just the degree of airflow limitation. However the assumption that symptoms do not equate to risk appears to be naïve, as groups B and C carry equally poor clinical outcomes though for different reasons. The latter may explain why group B patients are those most likely to switch group over time.

\section{Acknowledgements}

Authors thank all participants for their willingness to contribute to advance medical science in the field of COPD.

The principal investigators and centres participating in the ECLIPSE study are: Bulgaria: Y. Ivanov, Pleven; K. Kostov, Sofia. Canada: J. Bourbeau, Montreal; M. Fitzgerald, Vancouver; P. Hernández, Halifax; K. Killian, Hamilton; R. Levy, Vancouver; F. Maltais, Montreal; D. O’Donnell, Kingston. Czech Republic: J. Krepelka, Praha. Denmark: J. Vestbo, Hvidovre. The Netherlands: E. Wouters, Horn. New Zealand: D. Quinn, Wellington. Norway: P. Bakke, Bergen. Slovenia: M. Kosnik, Golnik. Spain: A. Agusti, Jaume Sauleda, Palma de Mallorca. Ukraine: Y. Feschenko, Kiev; V. Gavrisyuk, Kiev; L. Yashina. UK: W. MacNee, Edinburgh; D. Singh, Manchester; J. Wedzicha, London. USA: A. Anzueto, San Antonio, TX; S. Braman, Providence. RI; R. Casaburi, Torrance CA; B. Celli, Boston, MA; G. Giessel, Richmond, VA; M. Gotfried, Phoenix, AZ; G. Greenwald, Rancho Mirage, CA; N. Hanania, Houston, TX; D. Mahler, Lebanon, NH; B. Make, Denver, CO; S. Rennard, Omaha, NE; C. Rochester, New Haven, CT; P. Scanlon, Rochester, MN; D. Schuller, Omaha, NE; F. Sciurba, Pittsburg, PA; A. Sharafkhaneh, Houston, TX; T. Siler, St Charles, MO; E. Silverman, Boston, MA; A. Wanner, Miami, FL; R. Wise, Baltimore, MD; R. ZuWallack, Hartford, CT.

Authors' contributions. A. Agusti was a study investigator, developed the study protocol, served on the scientific committee, interpreted study data, developed the first draft of the manuscript, contributed to and reviewed drafts of the manuscript, and approved the final version of the manuscript; L.D. Edwards developed the study protocol, served on the steering committee, performed statistical analysis and interpreted data, contributed to and reviewed drafts of the manuscript, and approved the final version of the manuscript; B. Celli was a study investigator, developed the study protocol, served on the scientific committee, interpreted study data, contributed to and reviewed drafts of the manuscript, and approved the final version of the manuscript; W. MacNee was a study investigator, developed the study protocol, served on the Steering and Scientific (Chair) Committees, interpreted study data, contributed to and reviewed drafts of the manuscript, and approved the final version of the manuscript; P.M.A. Calverley developed the study protocol, served on the scientific committee, interpreted study data, contributed to and reviewed drafts of the manuscript, and approved the final version of the manuscript; H. Mullerova developed the study protocol, interpreted study data, contributed to and reviewed drafts of the manuscript, and approved the final version of the manuscript; D.A. Lomas developed the study protocol, served on the steering committee, interpreted study data, contributed to and reviewed drafts of the manuscript, and approved the final version of the manuscript; E. Wouters served on the scientific committee, developed the study protocol interpreted study data, contributed to and reviewed drafts of the manuscript, and approved the final version of the manuscript; P. Bakke was a study investigator, developed the study protocol, served on the steering committee, interpreted study data, contributed to and reviewed drafts of the manuscript, and approved the final version of the manuscript; S. Rennard was a study investigator, developed the study protocol, served on the scientific committee, interpreted study data, contributed to and reviewed drafts of the manuscript, and approved the final version of the manuscript; C. Crim developed the study protocol, served on the steering and scientific committees, interpreted study data, contributed to and reviewed drafts of the manuscript, and approved the final version of the manuscript; B.E. Miller interpreted data, contributed to and reviewed drafts of the manuscript, and approved the final version of the manuscript; H.O. Coxson developed the study protocol, served on the steering committee, interpreted study data, contributed to and reviewed drafts of the manuscript, and approved the final version of the manuscript; J.C. Yates developed the study protocol, served on the steering and scientific committees, interpreted study data, contributed to and reviewed drafts of the manuscript, and approved the final version of the manuscript; R. Tal-Singer developed the study protocol, served on the steering and scientific committees, interpreted study data, contributed to and reviewed drafts of the manuscript, and approved the final version of the manuscript; J. Vestbo was a study investigator, developed the study protocol, served on the steering committee, interpreted study data, contributed to and reviewed drafts of the manuscript, and approved the final version of the manuscript. The study sponsor did not place any restrictions with regard to statements made in the final version of the article. 
The Steering Committee included: P. Bakke (Norway), H. Coxson (Canada), L. Edwards (GlaxoSmithKline, USA), D. Lomas (UK), W. MacNee (UK), E. Silverman (USA), R. Tal-Singer (Co-chair, GlaxoSmithKline, USA), J. Vestbo (Cochair, Denmark), J. Yates (GlaxoSmithKline, USA).

The Scientific Committee included: A. Agusti (Spain), P. Calverley (UK), B. Celli (USA), C. Crim (GlaxoSmithKline, USA), B. Miller (GlaxoSmithKline, US), W. MacNee (Chair, UK), S. Rennard (USA), R. Tal-Singer (GlaxoSmithKline, USA), E. Wouters (The Netherlands).

\section{References}

Agusti A, Calverley P, Celli B, et al. Characterisation of COPD heterogeneity in the ECLIPSE cohort. Respir Res 2010; 11: 122.

2 Vestbo J, Hurd SS, Agusti AG, et al. Global strategy for the diagnosis, management and prevention of chronic obstructive pulmonary disease, GOLD executive summary. Am. J Respir Crit Care Med 2013; 187: 347-365.

3 Han MK, Mullerova H, Curran-Everett D, et al. GOLD 2011 disease severity classification in COPDGene: a prospective cohort study. Lancet Respi Med 2012; 1: 1-8.

4 Regan EA, Hokanson JE, Murphy JR, et al. Genetic epidemiology of COPD (COPDGene) study design. COPD 2010; 7: 32-43.

5 Lange P, Marott JL, Vestbo J, et al. Prediction of the clinical course of chronic obstructive pulmonary disease, using the new GOLD classification. Am J Respir Crit Care Med 2012; 186: 975-981.

6 Soriano JB, Alfajame I, Almagro P, et al. Distribution and prognostic validity of the new global initiative for chronic obstructive lung disease grading classification. Chest 2013; 143: 694-702.

7 Vestbo J, Anderson W, Coxson HO, et al. Evaluation of COPD longitudinally to identify predictive surrogate endpoints (ECLIPSE). Eur Respir J 2008; 31: 869-873.

8 Jones PW, Harding G, Berry P, et al. Development and first validation of the COPD Assessment Test. Eur Respir J 2009; 34: 648-654.

9 Standardization of spirometry, 1994 update. American Thoracic Society. Am J Respir Crit Care Med 1995; 152: $1107-1136$

10 ATS statement: guidelines for the six-minute walk test. Am J Respir Crit Care Med 2002; 166: 111-117.

11 Quanjer PH, Tammeling GJ, Cotes JE, et al. Lung volumes and forced ventilatory flows. Report Working Party Standardization of Lung Function Tests, European Community for Steel and Coal. Official Statement of the European Respiratory Society. Eur Respir J 1993; 6: Suppl. 16, 5-40.

12 Celli BR, Cote CG, Marin JM, et al. The body-mass index, airflow obstruction, dyspnea, and exercise capacity index in chronic obstructive pulmonary disease. N Eng J Med 2004; 350: 1005-1012.

13 Hurst JR, Vestbo J, Anzueto A, et al. Susceptibility to exacerbation in chronic obstructive pulmonary disease. $N$ Eng J Med 2010; 363: 1128-1138.

14 Agustí A, Edwards LD, Rennard SI, et al. Persistent systemic inflammation is associated with poor clinical outcomes in COPD: a novel phenotype. PLoS ONE 2012; 7: e37483.

15 Vestbo J, Edwards LD, Scanlon PD, et al. Changes in forced expiratory volume in 1 second over time in COPD. N Eng J Med 2011; 365: 1184-1192.

16 Jonckheere AR. A distribution-free k-sample test against ordered alternatives. Biometrika 1954; 41: 133-145.

17 Rabe KF, Hurd S, Anzueto A, et al. Global strategy for the diagnosis, management, and prevention of chronic obstructive pulmonary disease: GOLD executive summary. Am J Respir Crit Care Med 2007; 176: 532-555.

18 Gonen M, Heller G. Concordance probability and discriminatory power in proportional hazards regression. Biometrika 2005; 92: 965-970.

19 Mannino DM, Thorn D, Swensen A, et al. Prevalence and outcomes of diabetes, hypertension and cardiovascular disease in COPD. Eur Respir J 2008; 32: 962-969.

20 Casanova C, de Torres JP, Aguirre-Jaíme A, et al. The progression of chronic obstructive pulmonary disease is heterogeneous: the experience of the BODE cohort. Am J Respir Crit Care Mede 2011; 184: 1015-1021.

21 Nishimura M, Makita H, Nagai K, et al. Annual change in pulmonary function and clinical phenotype in chronic obstructive pulmonary disease. Am J Respir Crit Care Med 2012; 185: 44-52.

22 Postma D, Anzueto A, Calverley P, et al. A new perspective on optimal care for patients with COPD. Prim Care Respir J 2011; 20: 205-209. 